\title{
EFFECT OF COMMINUTION ON PARTICLE SHAPE AND SURFACE ROUGHNESS AND THEIR RELATION TO FLOTATION PROCESS: A REVIEW
}

\section{Mahmoud M. Ahmed}

Mining and Metallurgical Engineering Department, Faculty of Engineering, Assiut University, Assiut 71516, Egypt

\section{(Received April 22, 2009 Accepted May 11, 2009)}

The geometry of particles has an influence on the physical and chemical actions occurring on the particle surface within the technological processes. This illustrates the important of the exact geometrical evaluation of the particles by means of quantified particle parameters. These parameters can be used to differentiate between different materials or to correlate them with the different processes. The essential characteristics of the distinction of particles are size, shape and surface roughness.

Generally, particle shape and surface roughness can be considered as important parameters in the prediction of the behavior of particles individually or collectively. These parameters are of great importance to industries employing various materials in a powder form. These industries include many of the raw materials in civil and chemical engineering, pharmaceutical, mining industries, pigments, metals, ceramics, pills, foods, and industries interested with population of atmospheric dusts, smoke and grit. These parameters have not been currently reviewed satisfactory in the mineral processing field. Therefore, this review research is concerned with the different methods used to identify the particle shape and surface roughness and also to relate these parameters with the behavior of some mineral processing operations, especially comminution and flotation processes.

The surface roughness of mineral particles has influenced the fundamental processes of particle-bubble attachment and the other subprocesses in froth flotation. The contact angle is dependent on the surface roughness. The modification of the wettability due to surface roughness can be greatly enhanced in the fractal surface; that is the fractal surface will be supperrepellent/superwettable to a liquid when the contact angle is greater/less than $90^{\circ}$. Correlations were found between the shape properties, surface roughness values and wettability.

The dry grinding has produced relatively rough particle surfaces with a high concentration of microstructural defects while the wet grinding produced smoother cleaner surfaces. The dry ground samples exhibited more stable, higher loaded froths and faster flotation kinetics.

KEYWORDS: Particle shape, Surface roughness, Fractal, Comminution, Flotation, Wettability 


\section{NOMENCLATURE}

$\mathrm{B}_{\mathrm{M}} \quad$ maximum breadth of the projection of the profile on a direction at a right angle to that in which $\mathrm{L}_{\mathrm{M}}$ is measured

c constant

$\mathrm{D}_{\mathrm{A}} \quad$ particle diameter of a circle of equal area

$\mathrm{D}_{\mathrm{EM}} \quad$ diameter of embracing circle

D(Fmax.) Feret's diameter of the particle

$D_{i} \quad$ distance from the centroid of a particle to its edge

DX, DY, DP, DM particle diameters according to Feret in four different directions

F fractal dimension

Gl.B. soda-lime-glass ballotini

Gl.S. glass sand

Gr.B. ground ballotini

$\mathrm{g}_{\mathrm{ij}} \quad$ distance of the corner from the center of the maximum inscribed circle of radius $r_{i}$ for each of the three sections through the long, intermediate and short axises of the profile

LCNV circumference of the convex envelope of the particle

LENG circumference of the particle projection

$\mathrm{L}_{\mathrm{M}} \quad$ maximum length of the profile

$\mathrm{L}(\mathrm{r})$ particle perimeter length at step length ( $r$ )

$\mathrm{n}_{\text {cor. }} \quad$ number of concave corners of the particle

$\mathrm{n}_{\text {edg. }} \quad$ number of edges of the particle

Qz. quartizite

$\mathrm{r} \quad$ step length

$\mathrm{R}$ maximum inscribed radius of the particle

$\mathrm{R}_{\text {avg }}$ average particle radius (average over 180 two-degree increments)

$R_{i} \quad$ radii of curvature of $n_{\text {cor. }}$ (corners) of a projection or section of the particle

$\mathrm{B}_{\mathrm{ij}} \quad$ angle of each corner

\section{INTRODUCTION}

In addition to process variables, the processing of powder depends to a large extent on the materials (purity, structure, density, etc.), bulk properties, and the morphological characteristics (size, shape, texture, etc.). Materials characteristics are well understood and almost completely known since chemistry and physics of the material are highly developed areas but the same is not the case with the morphological characteristics. It is an underdeveloped and underexplored area, and unequivocal and unambiguous definitions of some of the morphological characteristics like particle shape, size, and surface roughness on which the bulk properties are strongly depended are yet to be evolved [1].

The geometry of particles has a pronounced influence on the physical and chemical actions occurring on the particle surface during the technological processes. This illustrates the importance of the exact geometrical evaluation of the particles by means of quantified particle parameters. These parameters can be used to differentiate between different materials or to correlate them with the different processes. The essential characteristics of the distinction of particles are size, shape and surface roughness [2]. Particle shape is being recognized as an important parameter in the prediction of the behavior of particles and powders influencing the manner in which they break, react, sinter, float, agglomerate and fluidize [3]. The distribution of particle sizes and shapes are of great importance to industries employing various materials in a 
powder form [4]. These include many of the raw materials in civil and chemical engineering, pharmaceutical, mining industries, pigments, metals, ceramics, pills, foods, and industries interested with population of atmospheric dusts, smoke and grit [5].

The first procedure developed, for qualitatively describing the shape of a fine particle, was carried out by measuring simple linear parameters such as the length, breadth and width. The ratios of these dimensions were then used as coefficients to describe the shape dependent property [6]. The methods used to assess the particle characteristics range from very simple (commonly used approaches) such as the determination of aspect ratio, elongation ratio or circularity to very modern methods such as the use of Fourier analysis, delta analysis and fractal geometry [1,7-8]. The development of automated microscope techniques linked to computer data-processing systems (image analysis techniques) has facilitated the possibility of using the methods of specifying the shape or surface roughness of a profile by mathematical procedures [8]. Development of these methods may give a way to optimize the mineral processing flow sheets not only on the basis of particle size distribution but also by the influence of combining the particle fractal properties [9].

Particle shape and surface roughness are considered as two of many factors which influence flotation particularly, and which may be less important than particle hydrophobicity and particle size.

Interaction between a solid particle and a gas-liquid interface controls the efficiency of the flotation process and has been considered in detail $[17,47]$. The bubble-particle interaction involves many stages, which are commonly referred to as the before-contact and after-contact interactions [10]. The before-contact bubbleparticle interaction is strongly affected by the long-range hydrodynamics when the bubble and the particle are far from each other whereas is controlled by interfacial physics e.g. interfacial dynamics, capillarity and colloidal interaction if they are close enough.

The particle shape has a significant influence on flotation effectiveness. Many researchers have studied this relation qualitatively [11-15]. Few researchers have studied the effect of the surface roughness on the flotation process [9,16-20]. The surface roughness of mineral particles has long been known to influence the fundamental processes of particle-bubble attachment and the other sub-processes in froth flotation.

Ahmed, M.M. [18-21] has illustrated an approach to study the quantitative effect of the surface roughness and shape of particles on the detachment process of particles from the air-liquid-interface by means of a centrifuge technique and also on their floatability (under ideal conditions) using a modified Hallimond tube. It can be concluded that the roughness of the solid surface has a decisive influence on the flotation effectiveness [9]. It can be decided that the higher the surface roughness, the stronger is the detachment force required to separate the particle from the bubble and hence a higher flotation recovery is obtained [18-19].

In froth flotation, the probability of flotation and hence rate of flotation is directly determined by three micro-processes: collision, adhesion and detachment [22]. To obtain an improved understanding of the complete flotation process, it is necessary to analysis these three micro-processes quantitatively. The ease with which the 
particles can be attached to the bubble and the strength of detachment are considered very important when the effectiveness of a flotation process is studied.

The differences in the values of detachment forces and subsequently flotation effectiveness were markedly clear at the different degrees of hydrophobicity for the same surface roughness. In addition, the differences in the values of detachment forces increased with increasing of the surface roughness. While at the lowest roughness, the difference in the value of detachment force between the lowest and height hydrophobicity does not exceed $1.5^{*} 10^{-5} \mathrm{~N}$, it was found that this difference reached to $7.1 * 10^{-5} \mathrm{~N}$ at the highest roughness. This behavior may be attributed to the chemical effects caused by adsorption of the silane films on the surfaces having different roughness. A surface with a lower roughness has a smaller surface area in compared with the surface of higher roughness and therefore it reaches a maximum surface coverage at a lower methylation time (lower contact angle) [18-19].

The effect of dry and wet grinding on the flotation of complex sulfide ores has been investigated [23]. The dry ground samples had relatively rough particle surfaces with a high concentration of microstructural defects while the wet ground samples had smoother cleaner surfaces. The dry ground samples exhibited more stable, higher loaded froths and faster flotation kinetics owing to the activated particle surfaces. Moreover, by combining dry and wet grinding the kinetics, as well as, the final grades and recoveries of the sulfides could be improved. In the flotation of sand the dry ground sand showed significantly faster flotation kinetics and a slightly higher flotation yield than the wet ground sand. This demonstrates that dry milling could improve flotation kinetics owing to the higher energy adsorbed in the particles.

In predicting the behavior of particles individually or collectively, particles shape and surface roughness are considered important parameters. These parameters have not been currently reviewed in the mineral processing operations satisfactory. Therefore, this review article is dominated by the different methods used to identify the particle shape and surface roughness and also to relate these parameters with the behavior of some mineral processing operations, particularly comminution and flotation processes.

\section{METHODS OF IDENTIFYING THE SHAPE AND SURFACE ROUGHNESS OF PARTICLES}

Particle shape is one of the most difficult problems and there is no general shape factor available which clearly differentiates between all possible kinds of shape [7]. The development of the automated microscope techniques linked to computer dataprocessing systems (image analysis techniques) has facilitated the possibility of using the methods of specifying the shape or surface roughness of a particle profile by mathematical procedures [8]. In this case, a three dimensional particle is projected in a plane to obtain two-dimensional closed curve which is taken to describe the particle shape. The methods used to assess the particle shape and surface roughness range from very simple (commonly used approaches) such as the determination of aspect ratio, elongation ratio or circularity to very modern methods such as the use of Fourier analysis, delta analysis and fractal geometry [1,7-8]. These methods are reviewed in the following sections: 


\subsection{Verbal Description}

This is the simplest method used for characterizing the particle shape. Words like spherical, disc, blade, rod, granular, rounded, angular, isometric, irregular, nodular, crystalline, oblong, acicular, fibrous, dendritic and flaky have been used to classify the particles into different shape groups. Although this identification corresponds well with the human ideas and his imagination, it is however quite subjective [1,24]. In many industries, an old system of separating particles based on their shapes is a vibrating table. In this device flat, elongated or needle like particles may be separated from rounded particles by flowing the particles over a titled table. The eccentric vibrating motion of the table drives all particles laterally along the table, however, rounded particles roll down the inclined side of the table at right angles to the motion of the flat particles. Parallel groves in the table may be used to keep acicular particles from rolling while the rounded chunky particles do roll [8].

\subsection{Mathematical Assessment of Shape and Surface Roughness of a Particle}

\subsubsection{Shape factors}

-Flatness ratio and elongation ratio [1,25]:

$$
\begin{aligned}
& \text { Flatnessratio }=\frac{\text { breadth }(B)}{\text { thickness }(T)} \\
& \text { Elongation ratio }=\frac{\text { length }(\mathrm{L})}{\text { breadth }(\mathrm{B})}
\end{aligned}
$$

- Circularity [1]:

$$
\text { Circularity }=\frac{\text { circumfere nce of a circle of the same area as the particle }}{\text { circumfere nce of theparticle }}
$$

- Factor of circularity (FK) [26]:

$$
\mathrm{FK}=\frac{\mathrm{D}_{\mathrm{A}}}{\mathrm{D}(\text { Fmax })}
$$

Feret's diameter, D(Fmax.) is defined as the maximum static length of particle projection derived from the co-ordinates of the projected area in one specified direction of measurement.

Factor of circularity characterizes the deviation from the circular shape. It has a value of 1.0 for the spherical particles and its value is less than 1.0 for the other particle shapes.

- Sphericity [1]:

$$
\text { Sphericity }=\frac{\text { surface area of a sphere of thesame volumeas the particle }}{\text { actual surface area of the particle }}
$$


Circularity and sphericity are measures of the degree to which the shape of a particle approaches to a circle and a sphere, respectively.

- External compactness [27]:

$$
\text { External compactness }=\left(\frac{\text { diameter of a circle of equal area }}{\text { diameter of embracing circle }}\right)^{2}=\left(\frac{\mathrm{D}_{\mathrm{A}}}{\mathrm{D}_{\mathrm{Em}}}\right)^{2}
$$

- Chunkiness [27-28]:

$$
\text { Chunkiness }=\frac{\mathrm{B}_{\mathrm{m}}}{\mathrm{L}_{\mathrm{m}}}
$$

- Aspect ratio [28]:

$$
\text { Aspect ratio }=\frac{\mathrm{L}_{m}}{\mathrm{~B}_{\mathrm{m}}}
$$

\subsubsection{Surface roughness factors}

- Roundness [1]:

$$
\text { Roundness }=\frac{\sum_{\mathrm{i}=1}^{\mathrm{p}} \mathrm{R}_{\mathrm{i}} / \mathrm{n}_{\text {cor }}}{\mathrm{R}}
$$

- Angularity [1]:

$$
\text { Angularity }=\sum_{i=1}^{3} \sum_{j=1}^{n}\left(180-\beta_{i j}\right) \cdot \frac{g_{i j}}{r_{i}}
$$

- Jaggedness [25]:

$$
\text { Jaggedness }=\frac{180\left(1-\frac{D_{i}}{R_{a v g}}\right)}{180}
$$

- Factor of concavity (CONC) [29]:

$$
\mathrm{CONC}=\left[\frac{2 \cdot(\mathrm{DX}+\mathrm{DY})+\mathrm{DP}+\mathrm{DM}+8}{2 \cdot \mathrm{n}_{\text {edg. }}-\mathrm{n}_{\text {cor. }}}\right]-1
$$

This measure has a zero value for the convex particles and increases with increasing fracturing.

- Factor of convexity (FO) [24]:

$$
\mathrm{FO}=\frac{\mathrm{LENG}}{\mathrm{LCNV}}
$$




\subsection{Fractal Geometry}

In recent years, there has been a very rapid development of two innovative procedures for determining the shape and surface roughness of fine particles profiles. These are Fourier analysis and the fractal geometry [28]. Fractal geometry was introduced to the scientific community by Mandelbrot in 1977 [20]. He illustrated how natural or real physical boundaries, which cannot be adequately described by traditional or Euclidean geometry, can be described using fractal geometry. Fractal geometry can be applied for characterization of the real physical boundaries which have no tangent, i.e. curves that exhibit no differential functions [31]. Fractal dimension is an interesting feature proposed recently to characterize the surface roughness and self-similarity in a picture [6]. The practical significance of the fractal dimension is many times more than the other measures, i.e. it can be quantified through a range of measuring scales and is expressed with a simple scalar number. It is practically well suited to describe the highly re-entrant particles, which have sharp corners and indentations [32].

The basic methodology by which the fractal dimension of a projected profile can be determined is to measure the perimeter many times with different scales of measurements, i.e. at a series of step lengths or resolutions. Smooth particles have a constant perimeter while rough particles have increasing perimeter length with decreasing step length [8]. Particles, which display fractal behavior, observe the following exponential relationship [6,8,31,33-36]:

$$
\mathrm{L}(\mathrm{r})=\mathrm{c} \cdot \mathrm{r}^{(1-\mathrm{F})}
$$

With large step lengths, the particle is modeled as a polygon with just a few sides of equal lengths and with small step lengths, the particle is modeled as a polygon with several hundred sides of equal lengths. Decreasing the step length results in an increase in the length of the estimated perimeter.

Eq. 14 can be rewritten as follows:

$$
\log [\mathrm{L}(\mathrm{r})]=(1-\mathrm{F}) \cdot \log [\mathrm{r}]+\log [\mathrm{C}]
$$

By plotting $\log [\mathrm{L}(\mathrm{r})]$ against $\log [\mathrm{r}]$, the fractal dimension $(\mathrm{F})$ can be obtained from the absolute slope (1 - F) of the best-fitting line of data in the plot. Such graph is known as a Richardson plot as illustrated in Fig. 1 where $1.0 \leq \mathrm{F} \leq 2.0$. The fractal dimension value equal to 1.0 is related to an Euclidean profile while the Brownian movement will approximate to a fractal dimension value equal to 2.0. Large slopes on the Richardson-graph correspond to a strong fracturing and an irregularity surface. It is usual to normalize the step length and the corresponding estimated perimeter to the Feret's diameter of the particle as shown in Fig. 2.

For natural systems, several dimensions can arise at various step lengths. Usually for mineral fragments, two linear portions with two different slopes are observed on the Richardson plot through the step length range used which illustrates different irregularities at different step lengths (different resolutions) as shown in Fig. 3. The region at the smaller step lengths is known as the textural (surface roughness) region while the structural (shape) region is at the coarser step lengths representing the gross boundary of the particle [31-34,37-38]. A sharp "break point" between the 
structure and the texture is significant where it is suggested a rapid transition from one fractal domain to the other. In many cases there is a gradual transition.

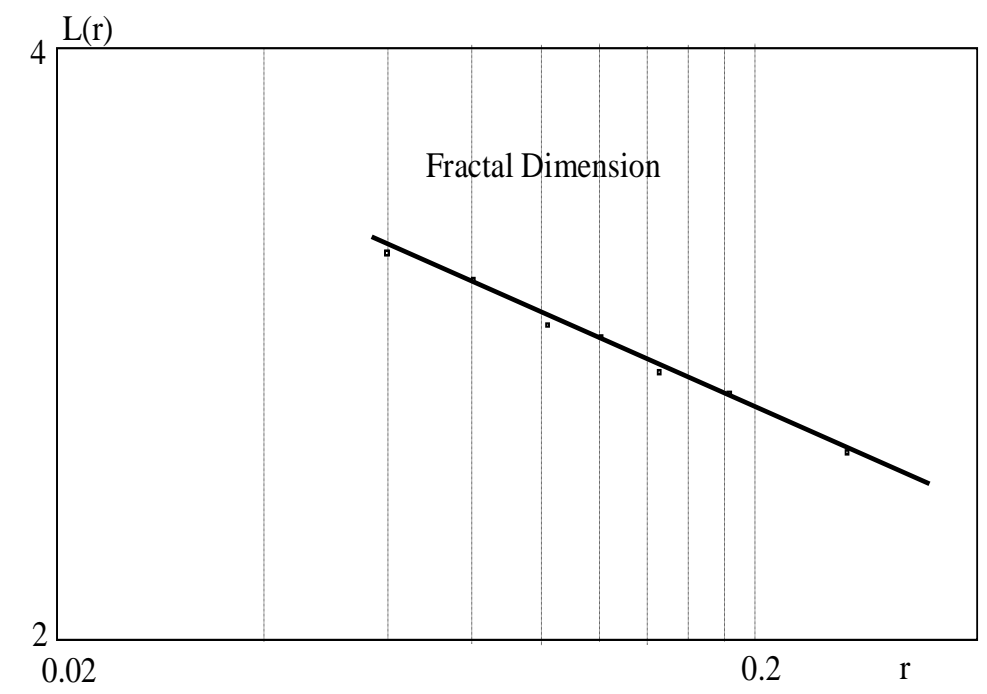

Fig. 1: A logarithmitic Richardson plot of a particle profile [after 6,8,31,33-36]

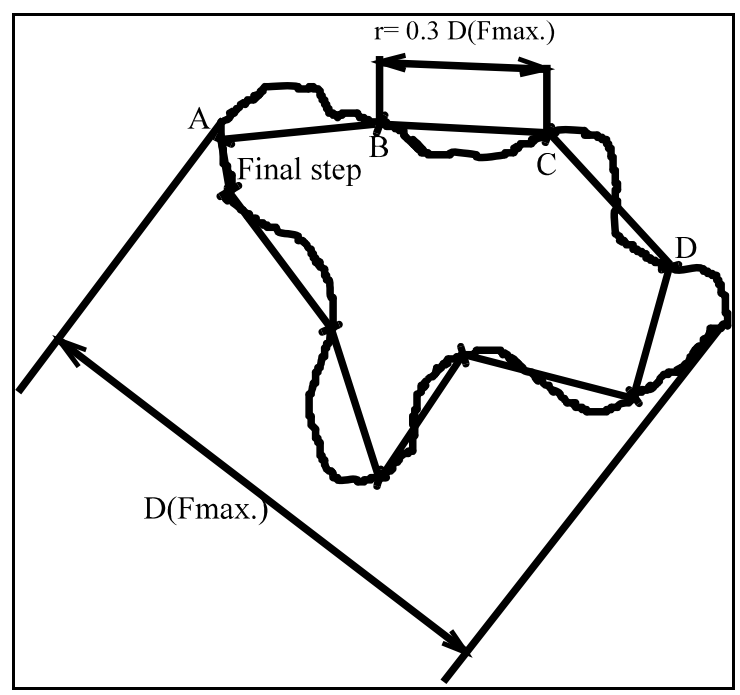

Fig. 2: A particle profile with a maximum step length of 0.3 of Feret's diameter [after

$$
\text { 6,8,31,33-36] }
$$

Kaye [39] and Birdi [40] also proved the above result in their investigations on carbon-black agglomerates. They decided that the shape of the particles is described by the structural fractal dimension and the surface roughness by the textural fractal dimension. A sharp "break point" between the structure and the texture is significant where it is suggested a rapid transition from one fractal domain to the other. In many cases there is a gradual transition. 


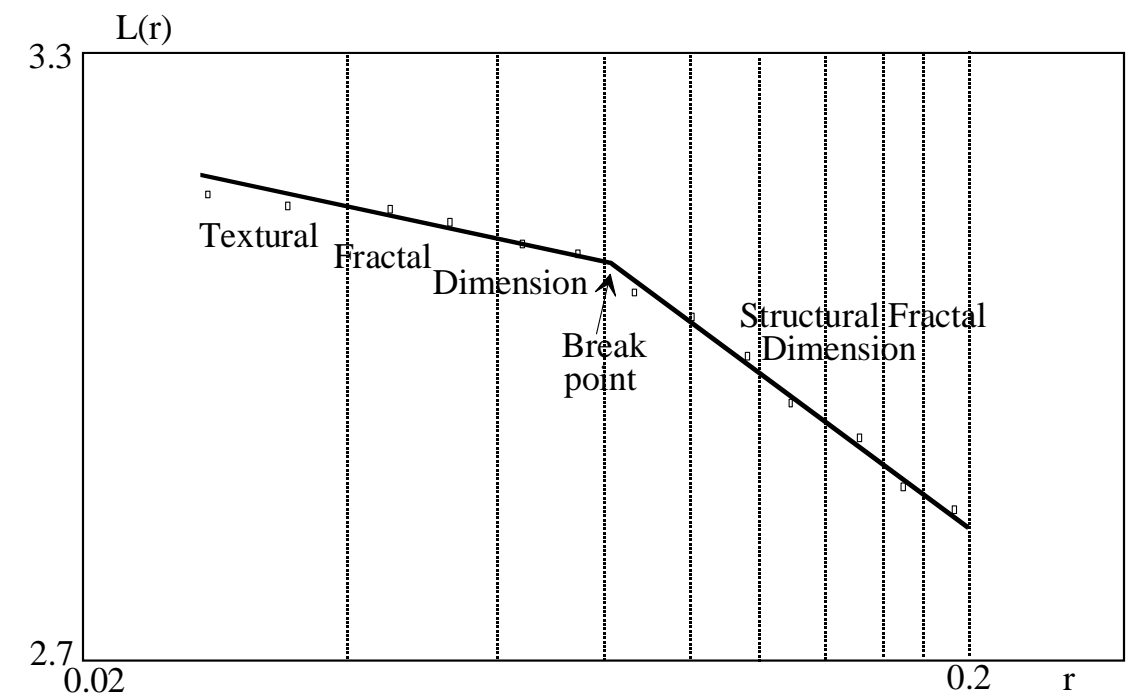

Fig. 3: Richardson plot showing the change in fractal dimension with the step length where the perimeter estimated and step lengths have been normalized with respect to Feret's diameter of the particle [after 31-34,37-38]

Graf [32] concluded that the range, number and dimension of fractal elements in a fine particle depend not only on the geometry of the particle but also on the resolution limits (step lengths) of the investigator. He listed four recommendations to remedy the problem of scale sensitivity:

1. Scales of Richardson diagrams must be made by using both the absolute and Feretnormalized measures. Absolute scales are useful because the particle geometry can be linked to the physical processes.

2. Select a constituent upper bound as the maximum step length. When very large step lengths are used, changes in the perimeter reflect the trends of simple polygons rather than the geometry of particle [41].

3. Use the largest possible resolution. Poor resolution, which results from the poor imaging or video limitations, can mask potentially important textural elements.

4. Make interpretations with the help of physical models and physical processes.

Various image analysis techniques have emerged over the last two decades. In these techniques, the three dimensions of the particle (or its projection in twodimensional direction) are actually measured [4]. Disadvantages of the twodimensional image analysis technique is that it gives no indication of the three dimensional geometry of the particle, where the technology is not generally available for three-dimensional digitization of the particle surfaces [36]. In earlier publications dealing with the fractal structure of fine particle boundaries, the experimental work was carried out manually. This was time consuming and the amount of investigative work, which could be undertaken in a given context, was limited [38]. But now several computer aided image analysis procedures have been reported. The most popular methods of determination the fractal dimension are structured walk technique, equipaced polygon technique, dilatation logic technique, the mosaic amalgamation technique and the line scans triangulation technique [3,38,41-44]. 


\subsection{Fourier Analysis}

One of the most popular methods for shape analysis is Fourier analysis which describes a given shape profile using Fourier coefficients [45]. In Fourier analysis, firstly a reference point at the center of profile is chosen. It is preferred to choose the centroid of the profile treated as a useful reference point. One then chooses a point on the periphery of the profile as the starting point for an exploration of the profile with a rotating vector. The maximum distance is then used as a normalizing factor to summarize the magnitude of the vector as it moves around the periphery of the profile with a uniform angular velocity to generate what looks like a waveform [46].

Two main procedures based on the Fourier analysis of a profile may be applied to the morphologic characterization of a particle [1]. The first method is based on Fixed Angular Boundary Sampling (FABS) [47] and the second is based on Fixed Boundary Segment Sampling (FBSS) [48].

\section{APPLICATION OF ATOMIC FORCE MICROSCOPY IN THE MEASUREMENT OF PARTICLE-BUBBLE INTERACTION AND SURFACE ROUGHNESS}

The atomic force microscopy (AFM) was first described in 1986 by Binnig and colleagues as a new technique for imaging the topology of surfaces to a high resolution [49]. Since then, AFM has enjoyed an increasingly ubiquitous role in the study of surface science, both as an imaging and surface characterization technique, and also as a mean of probing interactions and properties of single biomolecules by the application of force to these systems. AFM has a number of advantages over electron microscopy techniques, primarily the ability to make measurements in a fluid environment, as well as, the capability to directly measure interaction forces between surfaces [50]. It follows that an understanding of the nature and strength of the interactions between colloidal particles suspended in solution and air bubbles is of fundamental importance for developing new ways of increasing flotation efficiency and modulating specificity. Atomic force microscopy is one technique which is able to quantitatively measure interactions between single particles and interfacial boundaries. The AFM has been adapted for use in studying the forces involved in the attachment of single particles to bubbles in the laboratory. This allows the measurement of actual DLVO (Derjaguin, Landau, Vervey and Overbeek) forces and adhesive contacts to be measured under different conditions [50].

AFM was used to measure the contact angles of individual colloidal spheres (diameter $4.4 \mu \mathrm{m}$ ) and compare it to contact angles obtained on similarly prepared planar surfaces. For this purpose the particles were attached to atomic force microscope cantilevers. Then the force between the particle in aqueous medium and an air bubble was measured versus the distance. From the resulting force curves, it was obtained contact angles and detachment forces of single particles. Contact angles of gold coated silica particles were adjusted between $20^{\circ}$ and $100^{\circ}$ by self-assembling monolayers from different mixtures of undecanethiols and $\omega$-hydroxy undecanethiols from solution. In parallel, contact angles on flat gold surfaces prepared in the same way were determined by the sessile drop method. A systematic difference between contact angles measured with particles and on planar surfaces was observed. Results 
are discussed in terms of line tension of the three phase contact line. In addition, detachment forces were measured. Detachment forces were slightly higher than predicted from flotation theory. This might be caused by a pinning of the three phase contact line [51].

The AFM probe technique was used to measure hydrodynamic interaction forces between a solid sphere attached to an AFM cantilever and an air bubble placed on an AFM piezoelectric stage at different approach speeds. Interaction forces before the interfacial water film rupture, as well as, hydrophobicity of the particle can be established. In the case of hydrophobic spheres, strong attraction between the surfaces, leading to the rupture of the intervening water film and the attachment of the particle to the air bubble was observed. In the case of hydrophilic spheres, the rupture of the intervening water film and the attachment of the particle to the air bubble did not take place. Strong repulsive forces due to the hydrodynamic interaction were quantified. Theoretical hydrodynamic force showed agreement with experimental data for larger separation distances. Deviations at shorter distances are related to the deformation of air-water interface due to the particle approach, as well as, intermolecular and surface forces [52].

During recent years, many physicochemical phenomena in the bubble-particle attachment interaction have been investigated using atomic force microscopy (AFM) [53-56]. These research initiatives focused on the role of the interfacial properties, such as wettability of particles and electrical charges on the bubble and particle surfaces, and on the bubble-particle interaction. In particular, the intermolecular forces operating between the bubble and particle surfaces were determined. These studies also highlighted the experimental and theoretical difficulties of measurements and interpretation of the AFM data involving a deformable air-water interface. Despite numerous significant contributions to the theoretical analysis of interaction forces between a solid particle and a deformable interface, as well as, the deformation of this interface during the particle approach [57-58], there is still much we do not know. Nevertheless, significant progress has been made and AFM has been shown as a useful tool for study of particle-bubble interactions [52].

Surface properties like roughness, adsorption layers and surface chemistry strongly affect adhesion forces. Measurements of particle-particle and particle-surface interactions in the gas phase were carried out with an atomic force microscope (AFM). Special emphasis was given to a proper statistical treatment of the data. For modeling of particle adhesion, computer-assisted empirical potential methods were used. Parameters like adsorbed water and surface roughness were considered. Parameters for weak interactions from the Lifshitz theory and gas adsorption data were extracted. Adsorbing molecules can be used as probes to measure dispersive forces [59].

Reliable methods were made to characterize the surface roughness of solid particles for quality control and for finding the correlations with other properties. In this study, fractal analysis was used to describe the surface roughness. Atomic force microscopy (AFM) was used to obtain three-dimensional surface profiles. The variation method was used to calculate fractal dimensions. Fractal dimensions of four granule samples, four powders, and two freeze-dried powders were measured. A computer-program was written to implement the variation method. The implementation was verified using the model surfaces generated by fractional Brownian motion. The fractal dimensions of most particles and granules were between 
2.1 and 2.2, and were independent of the scan size measured. The freeze-dried samples, however showed wide variation in the values of fractal dimension, which were dependent on the scan size. As scan size increased, the fractal dimension also increased up to 2.5. This variation method allowed calculation of reliable fractal dimensions of surface profiles obtained by AFM. The estimates are dependent on the algorithm and the digitized model size (i.e., number of data points of the measured surface profile) used. The fractal dimension is also a function of the observation scale i.e., the scan size) used in the profile measurement. The multi-fractal features and the scale-dependency of fractal dimension resulted from the artificial processes controlling the surface morphology [60].

To obtain additional information on the surface roughness of the studied samples, AFM scans were performed and root mean square (r.m.s.) roughness was determined at different lateral scales [61-62]. Sizes are defined corresponding to specific physical properties measured on generally irregular particles, while most sizing techniques assume particles are spherical in shape. Direct physical particle sizes can be determined with an atomic force microscope [63].

\section{RELATIONSHIP OF THE COMMINUTION PROCESS WITH THE PARTICLE SHAPE AND SURFACE ROUGHNESS}

Although many aspects of comminution have been reported, there have been relatively few papers devoted to the effects on particle shape and surface roughness. This area of powder technology has been neglected partly due to the difficulties in measuring the shape and surface roughness of particles and also because there are no universally accepted definitions of shape [45].

A preliminary work [31] carried out on the applicability of fractal geometry in describing the products of comminution events, a nickel sulphide ore was subjected to two comminution events, impact shattering and ball milling. The size distributions of impact sample product were less than $2000 \mu \mathrm{m}$ (median size $=250 \mu \mathrm{m}$ ). The maximum size of ball milling sample was less than $3500 \mu \mathrm{m}$ (median size $=80 \mu \mathrm{m}$ ). Fractal analysis was performed on samples of the resulting comminution products. With the impact sample (formed by ballistic disintegration) exhibiting particles having higher boundary fractal dimensions than the milled sample. The fractal dimension of the impact fragments increased with decreasing size, whereas the milled particles displayed a more complex distribution. This type of behavior may be due to abrasion acting on fragmented particles in the ball mill, smoothing off any protrusions. Also, it is possible that small amounts of crushing (compressive failure) may have occurred, from the trapping of small particles between two larger balls. It was observed that fragments produced by tensile fragmentation have a higher fractal dimension than those produced by compressive fragmentation [31].

The data was plotted on Gaussian probability graph paper, as shown in Fig. 4, where the ordinate represents the cumulative percentage of a fractal dimension equal to or less than a stated value. The fact that the fractal information gave straight lines on Gaussian probability paper was evidence that fractal dimensions, in a population of shattered rocks, were probably describable by a Gaussian distribution function. Also, different comminution types occupy different regions on this type of chart, as indicated 
by Fig. 4 and various charts could be constructed for different materials. The implications are that if one required a definite particle distribution, in fractal terms, then the mechanism of size reduction could be selected [31]. There is clear evidence to support the concept that the structural fractal dimension of a particle holds key information upon its formation dynamics.

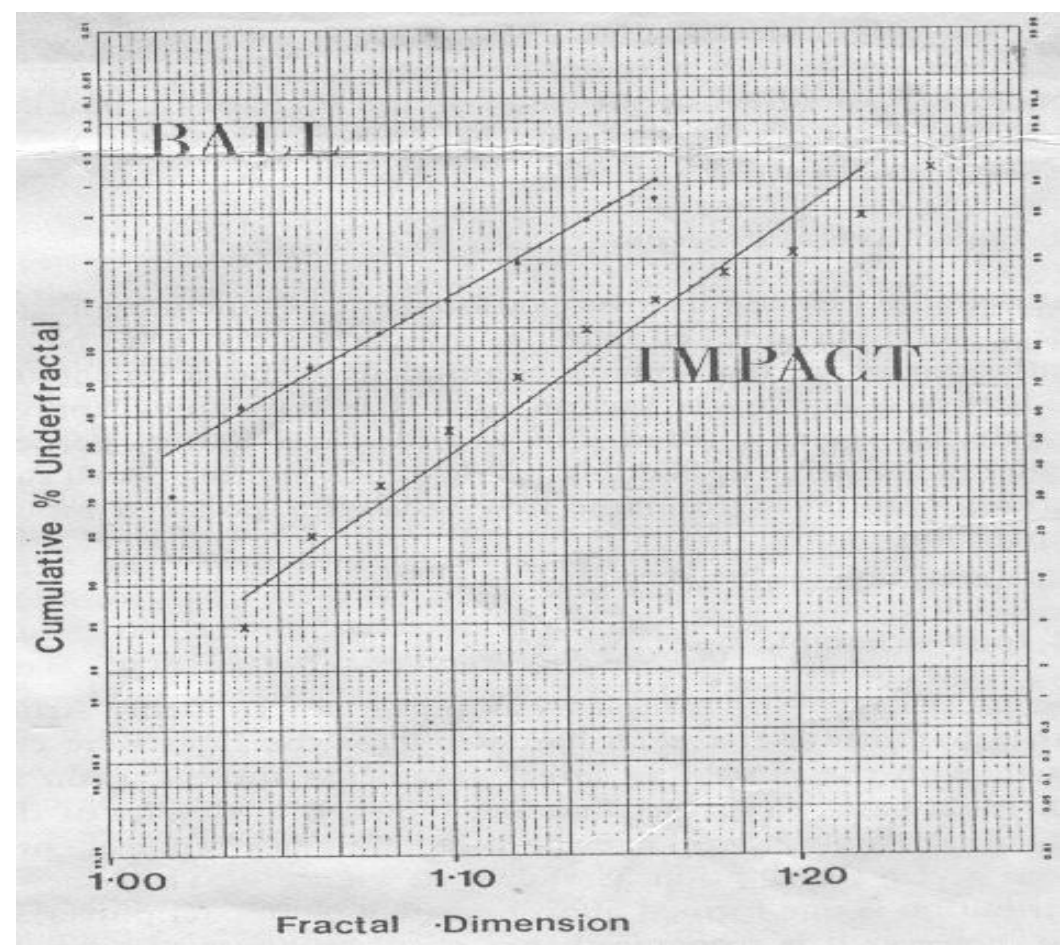

Fig. 4: Fractal distribution data illustrated on Gaussian probability graph paper a. impact sample product $\left(\mathrm{x}_{50}=250 \mu \mathrm{m}\right) \mathrm{b}$. ball mill product $\left(\mathrm{x}_{50}=80 \mu \mathrm{m}\right)$ [after 31]

Industrial materials, such as limestone, are suitable for flue gas desulphurization (FGD) owing to their physical properties and chemical contents. At present, more than $10 \mathrm{M}$ tones of limestone are milled per year in Europe for FGD purposes. A study [64] was performed to investigate if the type of pulverizer, used to comminute limestone, influenced its subsequent behavior. The three pulverizers under study were a laboratory hammer mill, crushing rolls with smooth surfaces and a batch laboratory ball mill. The failure mechanisms represented by these devices can be broadly categorized as single and/or double impact (hammer mill) and compression and shear failure (roll crusher). With the ball mill, size reduction mainly takes place by the impact of balls on the material, with either prior or subsequent abrasion (attrition) [64]. The studied sample of limestone consisted of $-14.0 \mathrm{~mm}$ size. It was pre-screened at the quarry, from which a $(-4.0+1.7) \mathrm{mm}$ size fraction was separated as a feed for the pulverizing devices. Samples of this fraction were comminuted by hammer milling, rolls crushing and ball milling as mentioned above. The resultant comminution products were screened at $38 \mu \mathrm{m}$ and the undersize was classified using a Warman cyclosizer (elutriation technique). Cone five on the cyclosizer of size $(-16.2+12.7) \mu \mathrm{m}$ was chosen as the size band for investigation [64]. 
The populations of the structural boundary fractal dimensions of the three sets of fragments are shown in Fig. 5. From this figure, it can be shown that the particles were Gaussianly distributed and the three distributions were found to be significantly different. This is further evidence to support the concept that the structural boundary fractal dimension of a particle holds key information upon its formation dynamics. On the other hand, simple geometric shape factors, i.e. circularity and aspect ratio did not detect any significant differences in the overall shape of the comminuted fragments. Variation in the limestone utilization was noted for the samples comminuted by the three different techniques, with the hammer-milled sample having the highest value because it has the highest boundary fractal population [34,64].

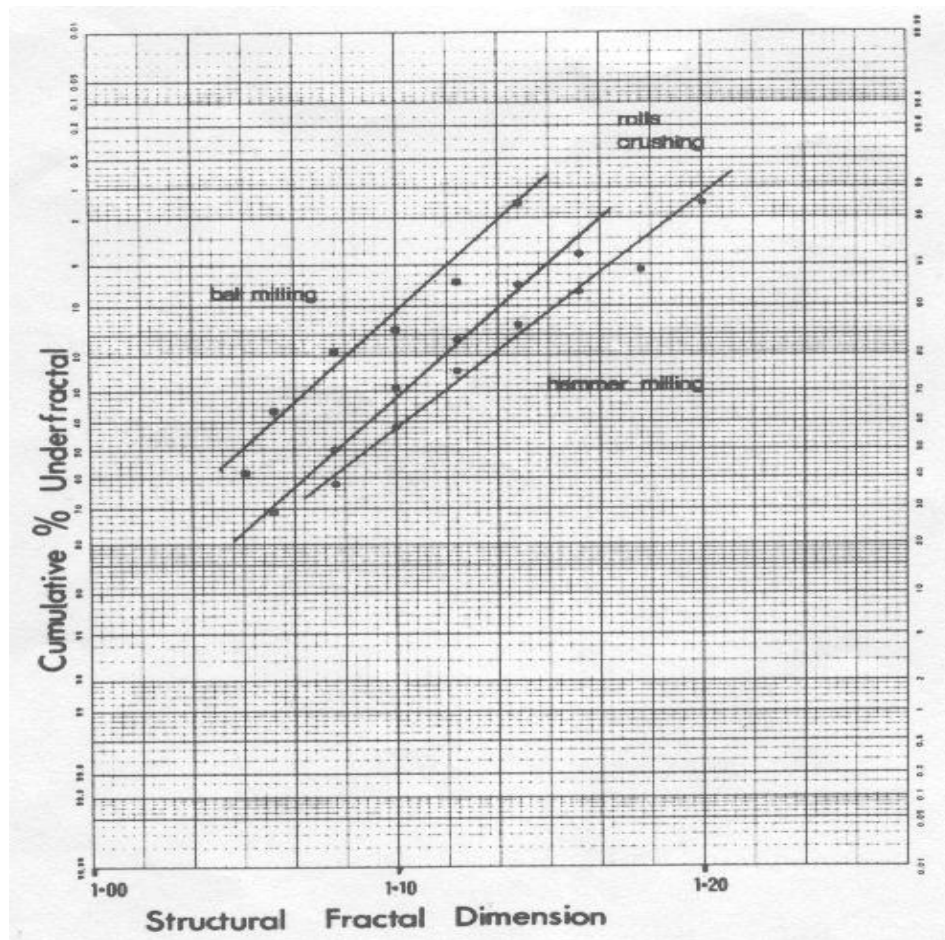

Fig. 5: Fractal distribution data, for the FGD limestone fragments, illustrated on a Gaussian probability graph paper $($ size $=-38 \mu \mathrm{m})$ [after 64]

Experimental investigations on the comminution of gibbsite in four different types of mills are presented. These included tests of dry grinding in a tumbling ball mill, a shaker bead mill and an air jet mill, as well as, tests of wet grinding in a tumbling ball mill and in a stirred bead mill. A quantitative characterization of the size and the shape of the ground product has been made in order to analyze the effect of the grinding stress on the properties of a complex agglomerate crystal such as gibbsite. The analysis allows us to determine the fragmentation route occurring in the mills. This has led to the identification of two fragmentation paths, involving different phenomena: rupture of joints, chipping and breakage according to the nature of the main stress (attrition or impact) imposed during comminution processes [65]. 


\section{EFFECT OF THE GRINDING TYPE ON THE FLOTATION PROCESS}

As well known, flotation differs from most of the separation processes by the fact that the conditions of separation directly depend on surface properties and therefore also on comminution process. The deformation mechanism in comminution has a great influence on the properties of mineral surfaces formed during grinding, because the geometrical heterogeneity of the surface determines the area reacting with flotation reagents. Moreover, the character of the interactions between the reagents and minerals is also dependent on the configuration of the surface [66].

The effect of dry and wet grinding on the flotation of complex sulfide ores from the Merensky Reef in South Africa was investigated. The sample was crushed in a jaw crusher and a rotary cone crusher to $100 \%$ passing $4 \mathrm{~mm}$. For comparative purposes, an oxide ore (sand) with a particle size of $3 \mathrm{~mm}$ was also investigated [23]. The rod mill was run at a constant speed of $80 \mathrm{rpm}$. Three samples were ground to approximately $60 \%$ passing $75 \mu \mathrm{m}$ under the conditions of dry milling, as well as, wet milling with a pulp concentrations of $66 \%$ and $90 \%$ solids. During dry milling, nitrogen was fed to the mill to drive off oxygen before milling started. The grinding results are as follows: Dry milling took $40 \mathrm{~min}$. to result $58.0 \%$ of $-75 \mu \mathrm{m}$. The dry ground sample had a specific surface area of $1.23 \mathrm{~m}^{2} / \mathrm{g}$. Wet milling at a pulp concentration of $66 \%$ solids took $30 \mathrm{~min}$. to ensure that $57.5 \%$ of the sample passed 75 $\mu \mathrm{m}$, the obtained product had a specific surface of $1.29 \mathrm{~m}^{2} / \mathrm{g}$. Wet milling at a pulp concentration of $90 \%$ solids took $36 \mathrm{~min}$. to give $58.5 \%$ of $-75 \mu \mathrm{m}$, this gave a specific surface of $1.25 \mathrm{~m}^{2} / \mathrm{g}$.

Topographical examination of the ground particle surfaces by scanning electron and atomic force microscopy showed that the dry ground samples had relatively rough particle surfaces with a high concentration of microstructural defects, while the wet ground samples had smoother, cleaner surfaces as shown in Fig. 6. Higher stresses were included in the particles in the denser slurry (90\% solids) and as a consequence some defects also appeared on these particle surfaces. When the same new surface formed, dry grinding consumed more energy than wet grinding. This means that the particles in dry grinding conserved more energy, some of which existed in the form of defects. These defects serve as active centers for spreading up particle dissolution and reagent adsorption [23].

As a result, the activated particle surfaces from the dry ground ore accelerated the dissolution of the particles, as well as, the adsorption of reagents onto the particle surfaces. The dry ground samples exhibited more stable, higher loaded froths and faster flotation kinetics, owing to the activated particle surfaces. High intensity conditioning of the dry ground ores prior to flotation could improve flotation by cleaning the particle surfaces through high shear force fields in the pulp. Moreover, by combining dry and wet grinding, the kinetics, as well as, the final grades and recoveries of the sulfides, could be improved as shown in Figs. 7 and 8 [23].

Sand samples were ground under dry and wet milling conditions. Dry milling took $50 \mathrm{~min}$. to ensure that $75 \%$ by mass of the sample passed $75 \mu \mathrm{m}$. The dry ground sample had a specific surface area of $1.68 \mathrm{~m}^{2} / \mathrm{g}$. Wet milling at a pulp concentration of $66 \%$ solids took $37 \mathrm{~min}$. to ensure that $75 \%$ by mass of the sample passed $75 \mu \mathrm{m}$. The wet ground sample had a specific surface of $1.76 \mathrm{~m}^{2} / \mathrm{g}$. Dry milling consumed 
approximately $25 \%$ more energy than wet milling to achieve more or less similar surface areas. Some of the energy adsorbed in the dry milling process was owed to particle deformation, which was stored in the form of surface defects on the particles. In addition, the particle surface was activated in the dry milling process. The wet ground sample had a smooth particle surface and few loose fine particles adsorbed onto the surface, while the dry ground sample had a rough particle surface and many small particles attached onto the surface. Some defects appeared on the particle surface in the dry ground sample, and these surface defects served as the active centers during reagent adsorption. As illustrated in Fig. 9, the dry ground sand showed significantly faster flotation kinetics and a slightly higher flotation yield than the wet ground sand. This demonstrates that dry milling could improve flotation kinetics, owing to the higher energy adsorbed in the particles [23].

(a)

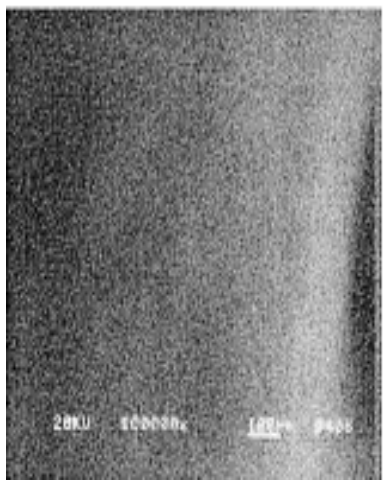

(b)

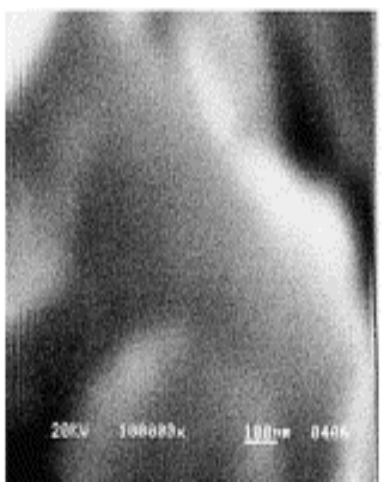

(c)

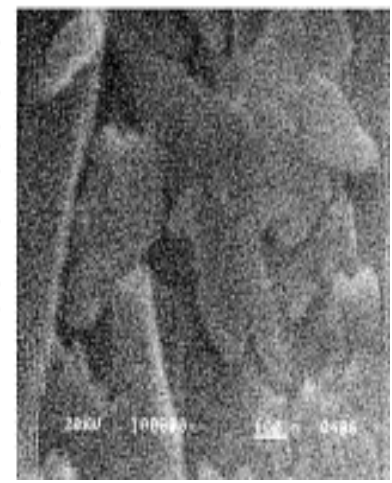

Fig. 6: Topographic of the sulphide particle surfaces by SEM: (a) wet grinding with $66 \%$ solids $\left(\%-75 \mu \mathrm{m}=58.0\right.$, surface area $\left.=1.23 \mathrm{~m}^{2} / \mathrm{g}\right)(\mathrm{b})$ wet grinding with $90 \%$ solids $\left(\%-75 \mu \mathrm{m}=57.5\right.$, surface area $\left.=1.29 \mathrm{~m}^{2} / \mathrm{g}\right)(\mathrm{c})$ dry grinding $(\%-75 \mu \mathrm{m}=58.5$, surface area $=1.25 \mathrm{~m}^{2} / \mathrm{g}$ ) [after 23]

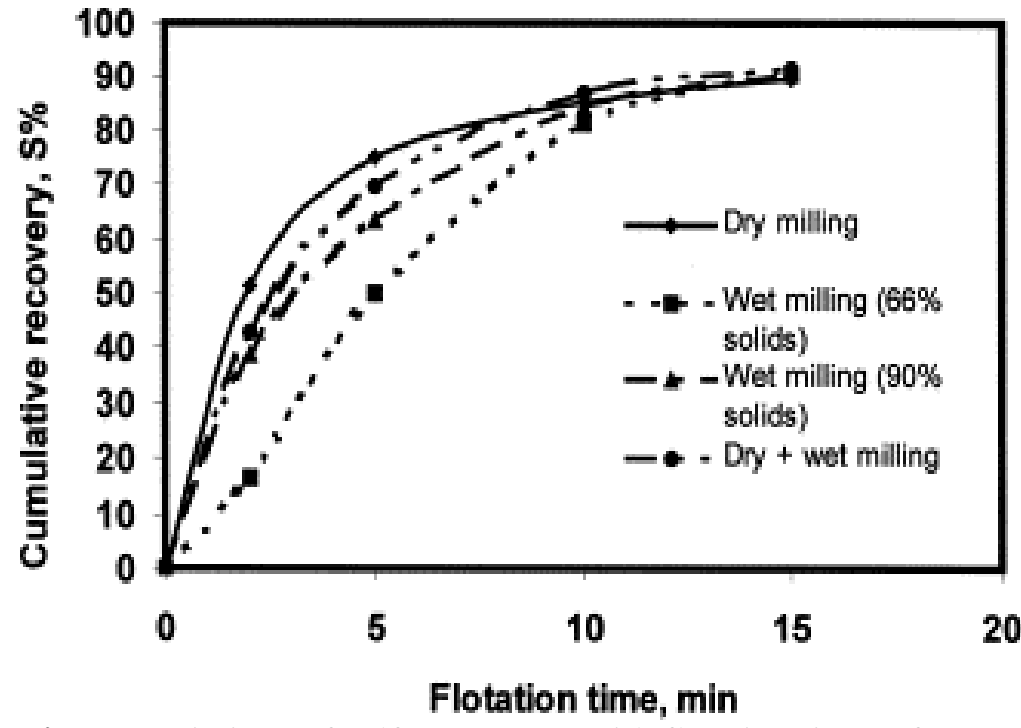

Fig. 7: Variations of sulfur recovery with flotation time [after 23] 


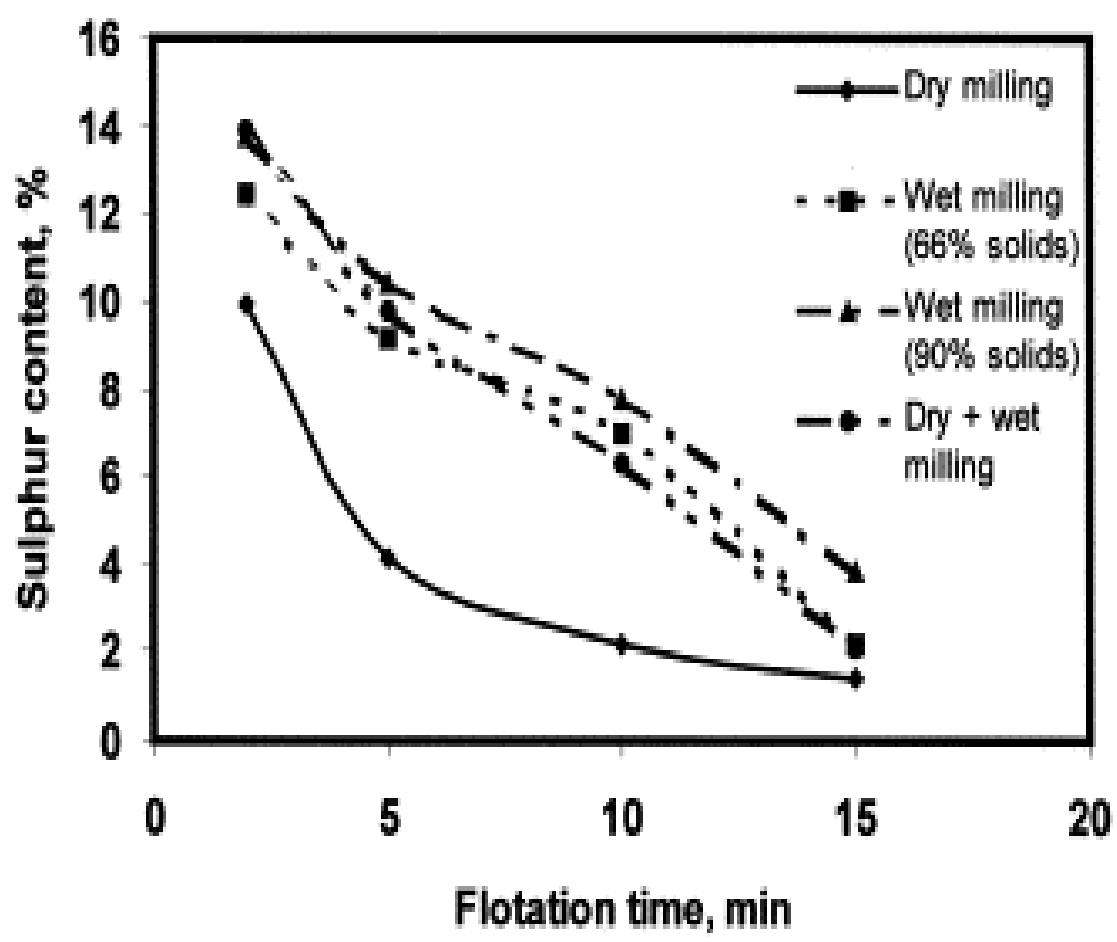

Fig. 8: Variations of sulfur content with flotation time [after 23]

In practice, dry milling of sulfide ores is complicated by the tendency of fine sulfides to oxidize in air. This is not the case with oxide ores, but the higher energy consumption has to be weighed against the enhanced flotation kinetics [23].

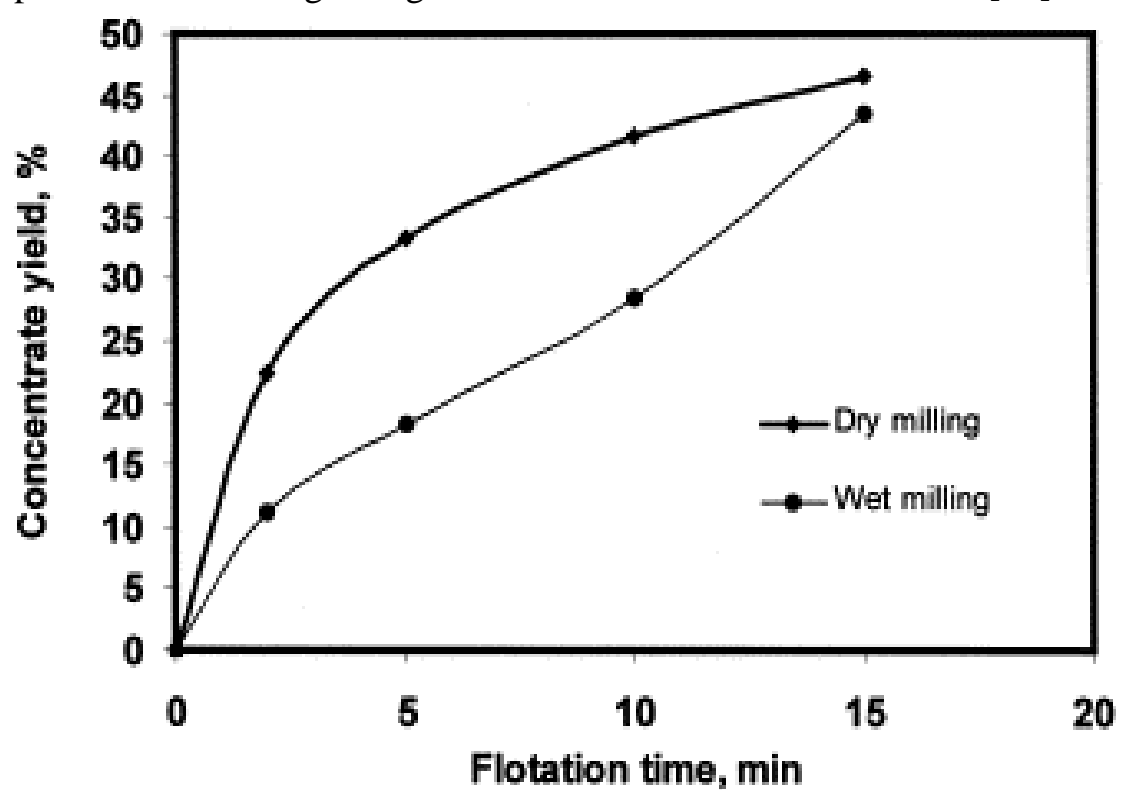

Fig. 9: Flotation result of sand samples under different milling environments: (a) wet milling with $66 \%$ solids $\left(\%-75 \mu \mathrm{m}=75.0\right.$, surface area $\left.=1.76 \mathrm{~m}^{2} / \mathrm{g}\right)(\mathrm{b})$ dry milling $\left(\%-75 \mu \mathrm{m}=75.0\right.$, surface area $\left.=1.68 \mathrm{~m}^{2} / \mathrm{g}\right)$ [after 23] 


\section{RELATIONSHIP OF THE PARTICLE SHAPE AND SURFACE ROUGHNESS WITH WETTABILITY}

Spreading of liquids on rough surfaces was studied experimentally and theoretically. Seven liquids, with viscosities spanning over a few orders of magnitude, were used on glass surfaces of five different values of average roughness, ranging from about 0.07 to about $40 \mu \mathrm{m}$ [67]. It is shown that the experimental data can be empirically correlated by a power law. In addition, a simple theoretical model was presented, which took into account the capillary flow of liquid into the roughness grooves. A Lucas-Washburn type of analysis for this flow was modified by taking into account the variation in the length of the capillary itself, due to the variation in the location of the edge of the drop that feeds the capillary flow. Results of the theoretical calculations were shown to be in good agreement with the experimental data [67]. The study of spreading on practical rough surfaces is difficult, because the problem of defining roughness is a complex one. Therefore, much work has been done on spreading on surfaces with well-defined grooves or roughness. Nonetheless, some measurements of the kinetics of spreading of liquids on practical rough surfaces were also made. These studies revealed that spreading of the liquid occurs by two modes: on top of the rough surface and inside the capillary grooves, which make up the roughness. The results were discussed in terms of power laws suitable for each of these spreading modes. However, no attempt has been made to develop a model that considers the two spreading modes simultaneously [67].

The process whereby a fluid comes in contact with a solid surface is very common in everyday life. At any interface between two phases (e.g. liquid-solid, liquid-gas, oil-water), the adjacent interfacial multimolecular layers are subjected to an unsymmetric force and consequently the interfacial tension is the net result of these forces [68]. The impact of surface roughness in wetting is beyond dispute. Quantification of the effects, however, is difficult and somewhat controversial. Much effort has been undertaken to study the equilibrium contact angle which can not be measured directly due to the existence of metastable states [69].

For rough surfaces, the meaning of a contact angle as a property is not clear from a force balance conceptual view. At sharp corners, the contact angle can be assumed as a range of values. Wenzel recognized this difficulty and approached the problem in terms of surface energies, rather than forces at an apparent and sometimes ambiguous line of contact. Wetting of a solid occurs only if there is a resulting decrease in free energy. The apparent equilibrium contact angle is then determined by an integrated surface-fluid-fluid interaction and not by conditions existing only at the point of contact [68].

It has been stated that the contact angle is dependent on the surface roughness [70]. The apparent contact angle for rough surfaces could be expressed in terms of that for smooth surfaces [68,70]. The modification of the wettability due to surface roughness can be greatly enhanced in the fractal surface; that is the fractal surface will be supperrepellent (superwettable) to a liquid when the contact angle is greater/less than $90^{\circ}[70]$.

In a paper published by Ulusoy and Yekeler [5], experimental studies to determine the shape properties, surface roughness and wettability of quartz mineral (a very common component of many raw materials) were performed for the products of 
ball, rod and autogenous mills. Shape properties have been stated in terms of shape descriptors such as elongation, flatness, roundness and relative width by measuring on the projections of particles using scanning electron microscope (SEM). Surface roughness values have been determined on the pelleted surfaces of the ground powders. The wettability characteristics (critical surface tension of wetting) of quartz mineral were determined by microflotation technique using the EMDEE MicroFLOT test tube. Finally, some correlations were found between the shape properties, surface roughness values and wettability. The results have shown that the critical surface tension of wetting values increase with increasing roughness and roundness, i.e. elongated and smooth particles having lower critical surface tension of wetting values indicated more hydrophobicity [5].

A new method for the measurement of apparent contact angles at the global energy minimum on real surfaces has been developed [71]. The method consists of vibrating the surface, taking top-view pictures of the drop, monitoring the drop roundness, and calculating the contact angle from the drop diameter and weight. The use of the new method has been demonstrated for various rough surfaces, all having the same surface chemistry. In order to establish the optimal vibration conditions, the proper ranges for the system parameters (i.e., drop volume, vibration time, frequency of vibration, and amplitude of vibration) were determined. The reliability of the method has been demonstrated by the fact that the ideal contact angles of all surfaces, as calculated from the Wenzel equation using the measured apparent contact angles, came out to be practically identical. This ideal contact angle has been compared with three methods of calculation from values of advancing and receding contact angles [71].

In many systems there exists a contact angle hysteresis between an advancing and receding contact angle, i.e. a different contact angle may be measured depending upon whether the three phase contact (TPC) line is advancing or receding over a particle surface. The reason for this hysteresis is generally ascribed to roughness and heterogeneity of the surface chemistry, although other factors may be of importance, such as polymer coatings, etc. [50].

\section{EFFECT OF PARTICLE SHAPE AND SURFACE ROUGHNESS ON THE FLOTATION EFFECTIVENESS}

The particle shape and its surface roughness have significant effects on the flotation behavior. Many researchers have studied these phenomena qualitatively [11-17], but few researchers [9] studied these phenomena quantitatively due to the lack of techniques required to measure them as quantified parameters. Ramesh and Somasundaran [72] decided that the shape of the particle does have a substantial effect on the force required to detach the particles from the gas-liquid interface. They found that homogeneous particles with a single specific geometry would yield a single value for the detachment force, but a mixture of particles (contact angle being the same for all the particles) having a variety of shapes would yield a distribution of detachment forces. Burstein et al. [9] showed that the flotation recoveries of non-spherical particles are higher than that of spherical one. A possible explanation of this result might be attributed to the increase of surface of non-spherical particles which leads to 
more adsorption of surfactant and hence increasing flotation recovery. This result was in a conflict with the result of Varbanov [10] who concluded that, with regard to practical flotation, no major differences could be inferred between the behaviors of spherical and non-spherical particles.

Schmidt and Berg [14] investigated the role of particle shape on flotation using model spheres and discs. Their results revealed that spheres float better than discs or plate-shaped. Spheres are deflected away from bubble by flow but usually attach if they contact the bubble surface. Discs often colloid with the bubble edge-on and immediately bounce off and seldom attach to the bubble due to the very short contact time. Alternatively, discs turn to the side as they approach the bubble but seldom attach due to the large thin film drainage area. Schmidt and Berg in other publication [15] by using a hydrodynamic model concluded that discs or flat, plate-shaped particles in general behave differently than more spherically shaped particles. Large disc-tobubble ratios $(>0.1)$ were found always to yield greater collision efficiencies than those for equivalent spherical particles. They found also that large discs always have a much lower probability of attachment than equivalent spheres due to the higher tendency of discs to bounce off the bubble surface after collision. For smaller discs, the differences in attachment efficiency and flotation behavior between discs and spheres decreased and collision efficiency became the predominant step.

Wotruba in his Ph.D. thesis [73] studied the influence of corn shape on the floatability of zirconium and microlith particles in a pneumatic flotation cell using prismatic, spherical and ellipsoidal shapes. The most important conclusions of this thesis are that the corn shape has an essential influence on the floatability of zirconium. Spherical and ellipsoidal particles are less floatable than prismatic particles, independent of whether the spherical particles are rough or not. Reasons for this behavior are that the attachment process with bubble is more difficult for spherical particles as compared to the prismatic particles. The second reason is that the retreatment in the liquid phase causes easier wetting on spherical particles than on prismatic particles with edges. Noteworsly, the strong influence of the shape on the floatability occurs at the range of $(-63+40) \mu \mathrm{m}$, i.e. in a range where the influence of the turbulence on the stability of the particle/bubble aggregate is not critical.

Few scientists studied the effect of surface roughness on the flotation process [9,16-17]. Burstein et al. [9] concluded that an increase in the particle roughness (determined in fractal terms) causes significant intensification of the process of collapse of lamellae near particle surfaces and reduction of froth stability. Anfruns and Kitchener [16] revealed that the particle roughness ensures easy rupture of the wetting film even when the barrier is strengthened by the addition of low concentrations of surfactant or when the contact angle is reduced from $87^{\circ}$ to $30^{\circ}$.

Ducker et al. [13] have studied the flotation of glass ballotini and ground ballotini using a double-chained cationic surfactant. They concluded that there are large differences in the flotation properties of these two materials. They interpreted these differences to the difference in surface roughness between glass ballotini and ground ballotini and not to be due to the difference in their shape. Anfruns and Kitchener [16] interpreted the behavior of collision and capture of the angular and smooth particles. They showed that the strongly hydrophobic angular quartz particles were captured by bubbles with $100 \%$ efficiency whereas the capture efficiency of a 
strongly hydrophobic glass ballotini was very much lower. The $100 \%$ capture efficiency of angular particles was attributed to the jagged projections or asperities on the particle surface which are leading to a local thinning and rupture of the wetting film, i.e. the flotation rate was enhanced as comparison to the smooth surface of the glass ballotini. Anfruns and Kitchener [16] also observed, for smooth particles, that although the static contact angle was $30^{\circ}$, they were not captured at all. They concluded, with smooth particles, that the capture rate was more sensitive to the operation of electrical double-layer forces during the thinning of the liquid film.

Ahmed, M.M. [18-21] introduced an approach to study the quantitative effect of the surface roughness and shape of particles on the detachment process of particles from the air-liquid-interface by means of a centrifuge technique and also on their floatability (under ideal conditions) using a modified Hallimond tube. His study was carried out on four different materials (spherical soda-lime-glass ballotini, ground ballotini, glass sand and quartzite) of the same particle size fraction $(-315+250) \mu \mathrm{m}$. For identification of the surface roughness of the particles, three parameters were used as follows: Textural fractal dimension (Ft), Factor of convexity (FO) and Factor of concavity (CONC). The following parameters were of interest for characterizing the shape of particles: Structural fractal dimension (Fs) and Factor of circularity (FK). The values of studied parameters were obtained by means of the software IMAGE_C of the image processing. The characterization of particle geometry was made as a two dimensional analysis of the digitized particle projection on a slide in the position of the greatest stability. According to Kaye [74], the average structure is described with a sufficient precision at the position of the greatest stability of the particles. From the results, it was found that the four materials investigated were different in surface roughness of particles. On the contrary, the particle shape of ground ballotini and the quartzite are the same in comparison to the other materials investigated.

\subsection{Effect of the Surface Roughness of Particles on Their Floatability}

Nguyen et al. [75] have made a detailed analysis of the particle/bubble attachment. There are three microprocesses (or elementary steps) for the before-contact stage which may occur for successful particle/ bubble attachment:

1. Thinning of the intervening liquid film to a critical thickness where the film rupture begins,

2. Rupture of the intervening liquid film i.e. formation of three-phase contact of a critical wetting radius and,

3. Expansion of the three phase contact line from the critical radius to form a stable wetting perimeter.

Schulze et al. [39] have decided that in the technological flotation process, the three microprocesses mentioned above occur simultaneously. Therefore it is impossible to determine unambiguously from the temporal course and the efficiency of the total process which one of the microprocesses is significant in the course of the process. Consequently this problem must be solved by investigating the individual microprocesses separately.

Figure 10 illustrates the effect of the surface roughness of particles (quantified by the textural fractal dimension) on their flotation recovery at different degrees of hydrophobicity. From this figure, it can be seen that as the textural fractal dimension 
increased, the flotation recovery increased linearly at different degrees of hydrophobicity. Similar relationships between the surface roughness (quantified by the factor of convexity and the factor of concavity) and flotation recovery were also obtained $[18,20]$. From Fig. 10, it can be seen that the differences in values of flotation recovery between the roughest material (quartzite) and the smoothest one (soda-limeglass ballotini) ranged from $24 \%$ (at contact angle $=71^{\circ}$ ) to $33.5 \%$ (at contact angle $=$ $78^{\circ}$ ). This means that although the small differences in the values of textural fractal dimension of materials examined, very great significant differences in values of flotation recovery between the smoothest material and the roughest one was obtained. This assures that the effect of the surface roughness on the flotation recovery was significantly effective.

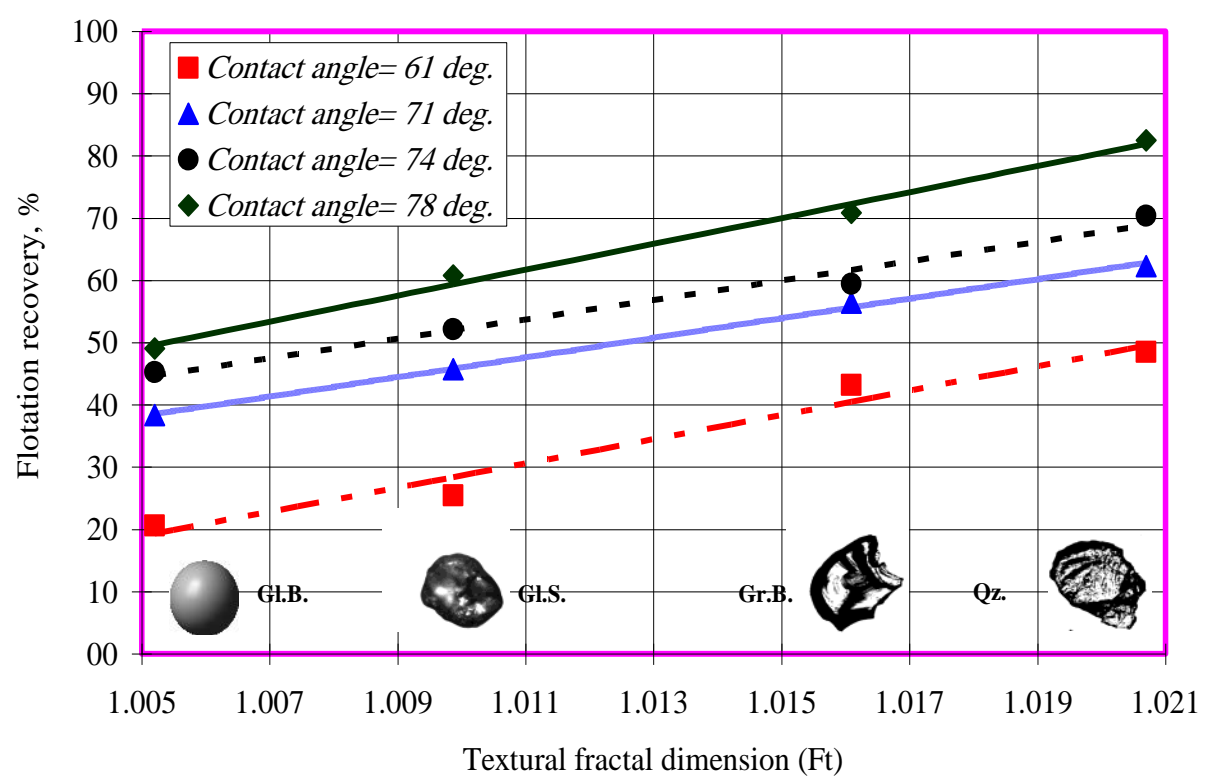

Fig. 10: Effect of the surface roughness of particles (quantified by the textural fractal dimension) on the flotation recovery at different degrees of hydrophobicity [after 18]

From the previous results, it can be concluded that the roughness has a significant effect on flotation recovery. This may be attributed to the fact that larger detachment forces required to separate the rough particles from bubble than to separate the smoother one. The larger detachment forces will indicate a strong adhesion force for the bubble/particle aggregates, which could reserve these aggregates to reach the float receiver and hence higher recovery is obtained [18-20].

It was decided that the surface roughness has an important role in the detachment process and then the effectiveness of flotation processes. This relationship was attributed to the larger values of three phase contact perimeter for rough particles more than smooth particles. Accordingly, a longer time, i.e. a larger detachment force is required to separate the particle from the gas-liquid interface for rough materials more than that for smooth materials [18-19]. This interpretation is equivalent to the process of expansion of three phase contact line on the surfaces of hydrophobic particles to attain the dewetting case. In this process, Nguyen et. al. [76] have observed 
experimentally that the time of three phase contact line expansion is a function of the central angle, i.e. the radius of three phase contact line. From this result it was concluded that a particle with a larger three phase contact radius needs a longer time to arrive to the dewetting case. This conclusion was exploited to support the results mentioned above. In the detachment experiments [18-19], a contraction of three phase contact line is occurring. This contraction on the particle surface is leading to attain to the wetting case and hence the particle is submerged into the liquid. This contraction needs a long time and then larger detachment forces (larger three phase contact perimeter) in the case of rough particles.

\subsection{Effect of the Particles Shape on Their Floatability}

Figure 11 shows the effect of the particle shape (quantified by the structural fractal dimension) on flotation recovery of the materials examined at different degrees of hydrophobicity. From this figure, it can be seen that as the structural fractal dimension increased from 1.020 to 1.043 , the flotation recovery slightly increased. Above this value, any increase in the structural fractal dimension causes significant increase in flotation recovery. This behavior is the same at all degrees of hydrophobicity [18,20]. Fig. 12 illustrates the effect of particle shape (quantified by the factor of circularity) on flotation recovery at different degrees of hydrophobicity. From this figure, it can be seen that as the particle shape increased (decreasing of the factor of circularity), the flotation recovery increased up to a certain point above which an excessive increase in the particle shape will result in a decrease in the final flotation recovery. It can also be noticed that the relationship between the particle shape and flotation recovery has the same behavior at all degrees of hydrophobicity [18,20].

Comparison of the results shown in Figures 11 and 12 displayed that there is a clear difference in the behavior of flotation recovery with the particle shape (if this shape was quantified by the structural fractal dimension or the factor of circularity). This means that the particle shape is not as effective as the surface roughness in controlling the flotation recovery. This result may also be ascertained from Fig. 11. In this figure, it can be observed that although the structural fractal dimensions of ground ballotini and quartzite are almost the same (these materials have the same shape), they have different flotation recoveries [18,20]. Figs. 11 and 12 showed also that the flotation recoveries of ground ballotini are higher than that of spherical ballotini at all degrees of hydrophobicity. A possible explanation of this result may be attributed to an increase in the particle surface with the rise of structural fractal dimension of ground ballotini leading to more adsorption of surfactant [9] and hence increasing in the flotation recovery [18-19]. 


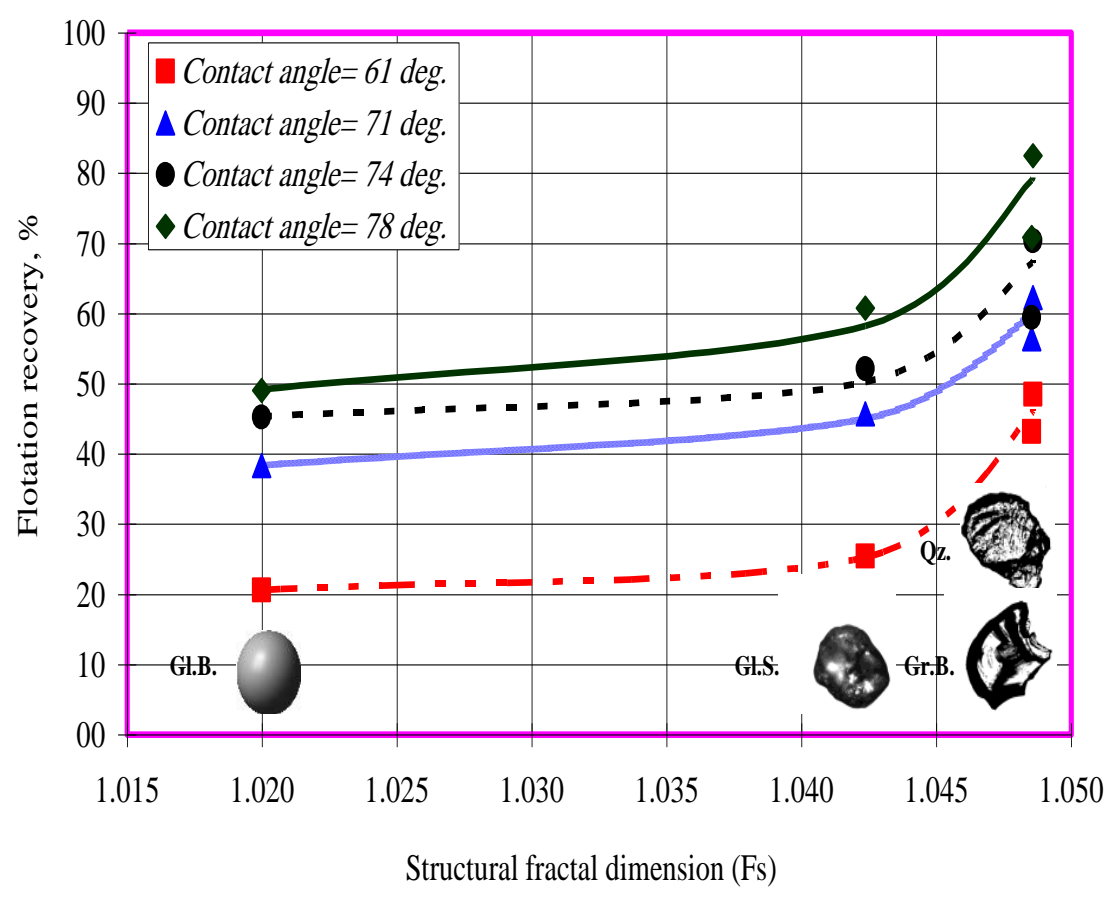

Fig. 11: Effect of the shape of particles (quantified by the structural fractal dimension) on the flotation recovery at different degrees of hydrophobicity [after 18]

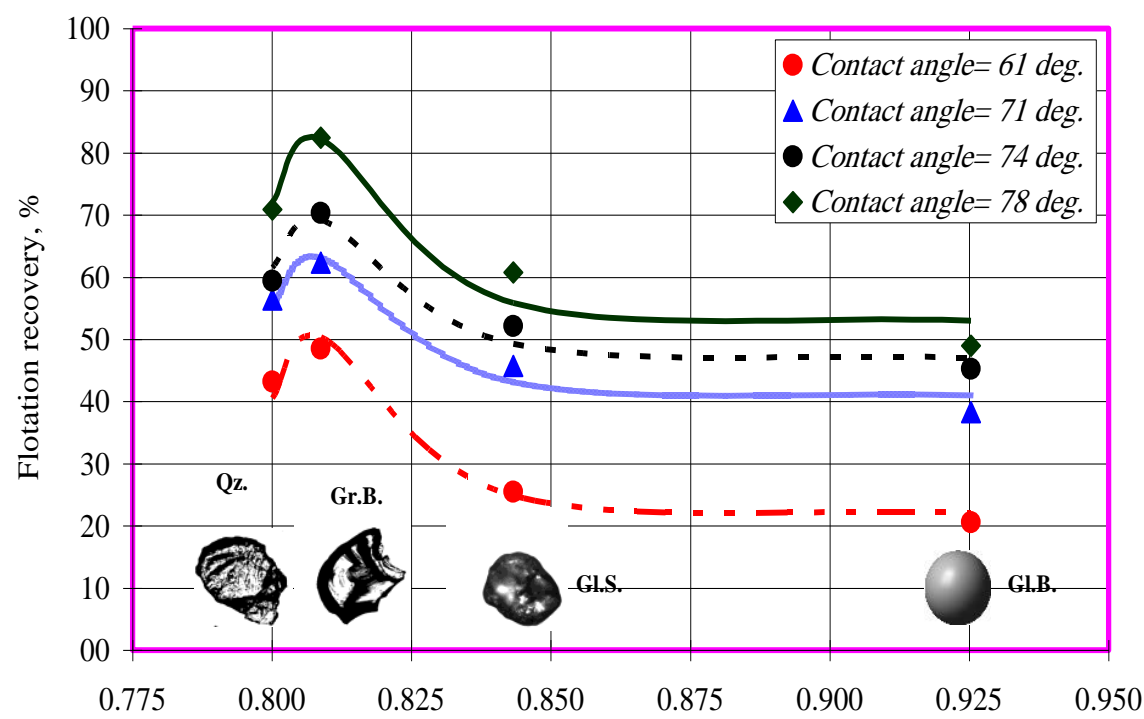

Factor of circularity (FK)

Fig. 12: Effect of the shape of particles (quantified by the factor of circularity) on the flotation recovery at different degrees of hydrophobicity [after 18] 


\section{SUMMARY}

1. Particle shape and surface roughness being recognized as important parameters in the prediction of the behavior of particles and powders influencing the manner in which they break, react, sinter, agglomerate, flow and fluidize.

2. The methods used to assess the particle shape and surface roughness range from very simple (commonly used approaches) such as the determination of aspect ratio, elongation ratio or circularity to very modern methods such as the use of Fourier analysis, delta analysis and fractal geometry.

3. The development of automated microscope techniques linked to computer dataprocessing systems (image analysis techniques) has facilitated the possibility of using the methods of specifying the shape or surface roughness of a profile by mathematical procedures.

4. Particles resulted from the impact crushing having higher boundary fractal dimensions than the rotary milled particles. The fractal dimension of the impact fragments increases with decreasing size whereas the milled particles displays a more complex distribution.

5. The under-fractal distributions of fragments suggest that for a particular material if a specific particle distribution is required, in fractal terms, the mechanism of size reduction could be selected.

6. The contact angle is dependent on the surface roughness. At sharp corners, the contact angle can be assumed as a range of values of angles. The apparent contact angle for rough surfaces could be expressed in terms of that for smooth surfaces.

7. The modification of the wettability due to surface roughness can be greatly enhanced in the fractal surface; that is the fractal surface will be supperrepellent (superwettable) to a liquid when the contact angle is greater/less than $90^{\circ}$.

8. The particle shape does not control clearly the flotation recovery, i.e. the surface roughness is responsible for the detachment process of particles, as well as, the final flotation recovery values more than its shape.

9. The dry ground samples exhibit more stable, higher loaded froths and faster flotation kinetics because the dry ground samples have relatively rough particle surfaces while the wet ground samples have smoother and cleaner surfaces.

\section{REFERENCES}

1. Singh, P. and Ramakrishnan, P., "Powder characterization by particle shape assessment", KONA, No. 14, pp. 16-30, (1996).

2. Castellini, C., Francini, C., Longobardi, G. and Pampaloni, E., "On-line characterization of the shape and size of particles", Part. Part. Syst. Charact., Vol. 10, pp. 7-10, (1993).

3. Clark, N.N., "Three techniques for implementing digital fractal analysis of particle shape", Powder Technology, Vol. 46, pp. 45-52, (1986).

4. Hurter, P. and Clark, N.N., "Persistence of polygonal harmonics as shape descriptors", Part. Part. Syst. Charact., Vol. 4, pp. 101-105, (1987). 
5. Ulusoy, U., Yekler, M. and Hicyilmaz, C., "Determination of the shape, morphological and wettability properties of quartz and their correlations", Minerals Engineering, Vol. 16, pp. 951-964, (2003).

6. Sarkar, N. and Chaudhuri, B.B., "An efficient differential box-counting approach to compute fractal dimension of image", IEEE Transactions on Systems, Man. and Cybernetics, Vol. 24, No. 1, January, (1994).

7. Podczeck, F., "A shape factor to assess the shape of particles using image analysis", Powder Technology, Vol. 93, pp. 47-53, (1997).

8. Meloy, T.P. and Williams, M.C., "Particle shape characterization, new and old methods: does a particle have shape", In: Challenges in Mineral Processing, Chapter 12, pp. 207-221, (1994).

9. Burstein, M.A., Hall, S.T. and Kornyshev, A.A., "A study of the effect of particle fractal geometry on flotation performance", Proc. Int. Miner. Process. Congr., Soc. Min. Metall. Explor., Littleton, Colorado, U.S.A., Vol. 3, pp. 77-80, (1995).

10. Scheludko, A., Radoev, B., Fabrikant, A., "On the theory of flotation: II. Adhesion of particles to bubbles", Annuaire de l'université de Sofia, Kliment Ochridski, Faculté de Chemie, Vol. 63, pp. 43-54, (1968/1969).

11. Varbanov, R., "Flotation of spherical particles", Trans. IMM, Sec. C, Vol. 93, pp. C6-C8, (1984).

12. Blake, P. and Ralston, J., "Particle size, surface coverage and flotation response", Colloids and Surfaces, Vol. 16, Issue No. 4, pp. 41-53, (1985).

13. Ducker, W.A., Pashley, R.M. and Ninham, B.W., "The flotation of quartz using a double-chained cationic surfactant", J. of Colloid and Interface Sci., Vol. 128, No. 1, pp. 66-75, (1989).

14. Schmidt, D.C. and Berg, J.C., "The effect of particle shape on the flotation of toner particles", Progress in Paper Recycling, February, pp. 67-77, (1996).

15. Schmidt, D.C. and Berg, J.C., "A preliminary hydrodynamic analysis of the flotation of disc-shaped toner particles", Progress in Paper Recycling, February, pp. 38-49, (1997).

16. Anfruns, J.F. and Kitchener, J.A., "Rate of capture of small particles in flotation", Trans. IMM, Sec. C, Vol. 86, pp. C9-C15, (1977).

17. Silverstein, M.S. and Breuer, O., "Wettability and flotation of etched ultra high molecular weight polyethylene fibres", Polymer, Vol. 34, No. 16, pp. 3421-3427, (1993).

18. Ahmed, M.M., "Fractal surfaces of particles and their floatability", Ph.D. Thesis, Assiut Univ., Assiut, Egypt, (1999).

19. Ahmed, M.M., Stechemesser, H., Mabrouk, S.A., Ibrahim, G.A., Tarshan, M.M., "Image analysis technique for determination of the size, structure and texture of fine particle profile", The 6th International Conference on Mining, Petroleum and Metallurgy, Vol. I-B, Mineral Processing, Fac. of Engng., Cairo Uni., Cairo, Egypt, February 20-24, pp. 40-56, (1999).

20. Stechemesser, H., Ahmed, M.M., "Flotation-shape and surface roughness", 50 Berg-und Huettenmaennischer Tag, Kolloquium 2, Sortierung der Abfaelle und Mineralischen Rohstoffe, Freiberg, Germany, 16 bis 18 Juni, pp. 120-131, (1999). 
21. Ahmed, M.M., Stechemesser, H., Mabrouk, S.A., Ibrahim, G.A., Tarshan, M.M., "The relationship between the surface roughness, shape and detachment force of particles from the liquid/gas interface using centrifuge method", 49 Bergund Huettenmaennischer Tag, Kolloquium 2, Partikeltechnologie, Freiberg, Germany, 18 und 19 Juni, pp. 207-223, (1998).

22. Trahar, W.J., Warren, L.J., "The floatability of very fine particles: a review", Int. J. Miner. Process, Vol. 3, pp. 103-131, (1976).

23. Feng, D. and Aldrich, C., "A comparison of the flotation of ore from the Merensky Reef after wet and dry grinding", Int. J. Mineral Process., Vol. 60, pp. 115-129, (2000).

24. Stechemesser, H., Partzscht, H. and Zobel, G., "A procedure for the characterization of shape and roughness of particles by means of digitized image analysis", Aufbereitungs Technik, Vol. 37, No. 9, pp. 422-431, (1996).

25. Broyles, D.A., Rimmer, H.W. and Adel, G.T., "Rapid shape analysis of crushed stone using image analysis", KONA, No. 14, pp. 130-137, (1996).

26. Stoyan, D., "Fraktale-Formen-Punktfelder: Methoden der Geometrie Statistik", Akademie Verlag, Berlin, (1993).

27. Kaye, B.H., "Fractal and shape index description of the shape and surface structure of metal powders ", Modern Developments in Powder Metallurgy, Hausner, H.H. (Ed.), Vol. 12, pp. 67-82, (1981).

28. Kaye, B.H., Clark, G.G. and Liu, Y., "Characterizing the structure of abrasive fineparticles", Part. Part. Syst. Charact., Vol.. 9, pp. 1-8, (1992).

29. IMAGE_C: Software to Image Analysing System "IMTRON 2000", IMTRONIC GmbH, Berlin, (1993).

30. Mandelbrot, B.B., "Fractals: Form, Chance and Dimension", W.H. Freeman, San Francisco, (1977).

31. Brown, G.J., Miles, N.J. and Hall, S.T., "Fractal characterization of pulverised materials", Part. Part. Syst. Charact., Vol. 10, pp. 1-6, (1993).

32. Graf, J.C., "The importance of resolution limits to the interpretation of fractal descriptions of fine particles", Powder Technology, Vol. 67, pp. 83-85, (1991).

33. Kaye, B.H., "The impact of fractal geometry on fineparticle characterization", An Invited Review at Particle Size Analysis Conference, Loughborough, pp. 300313, (1991).

34. Brown, G.J., Miles, N.J. and Hall, S.T., "Characterization of mineral powders by applied fractal geometry", Innovations in Mineral Processing, Sudbury, June, pp. 497-502, (1994).

35. Lee, C.T. and Chou, C.C., "Application of fractal geometry in quantitative characterization of aerosol morphology", Part. Part. Syst. Charact., Vol. 11, pp. 436-441, (1994).

36. Clark, N.N., "A new scheme for particle shape characterization based on fractal harmonics and fractal dimensions", Powder Technology, Vol. 51, pp. 243-249, (1987).

37. Kaye, B.H. and Clark, G.G., "Experimental characterization of fineparticle profiles exhibiting regions of various ruggedness", Part. Part. Syst. Charact., Vol. 6, pp. 1-12, (1989). 
38. Kaye, B.H., Clark, G.G., Leblanc, J.E. and Trottier, R.A., "Image analysis procedures for characterizing the fractal dimension of fineparticles", Part. Part. Syst. Charact., Vol. 4, pp. 63-66, (1987).

39. Kaye, B.H., "Specification of the ruggedness and/or texture of a fine particle profile by its fractal dimension", Powder Technology, Vol. 21, pp. 1-16, (1978).

40. Birdi, K.S., "Fractals in Chemistry, Geochemistry and Biophysics", Plenum Press, New York, (1993).

41. Kaye, B.H., "Fractal description of fineparticle systems", Particle Characterization in Technology, CRC Press, Boca Raton/Florida, Volume I, pp. 81100, (1984).

42. Kaye, B.H., Clark, G.G. and Kydar, Y., "Strategies for evaluating boundary fractal dimensions by computer aided image analysis", Part. Part. Syst. Charact., Vol. 11, pp. 411-417, (1994).

43. Schwarz, H. and Exner, H.E., "The implementation of the concept of fractal dimension on a semi-automatic image analyser", Powder Technology, Vol. 27, pp. 207-213, (1980).

44. Avnir, D., "The Fractal Approach to Heterogeneous Chemistry: Surfaces, Colloids, Polymers", John Wiley\&Sons, New York, (1989).

45. Kaya, E., Kumar, S.R. and Hogg, R., "Particle shape characterization using an image analysis technique", Proc. of the $6^{\text {th }}$ Int. Mineral Process. Symposium, Changing Scopes in Mineral Process., Kemal, Arslan, Akar\&Canbazoglu (eds), Balkema, Rotterdam, Turkey, pp. 47-52, Sept. 24-26, (1996).

46. Schulze, H.J., Radoev, B., Geidel, Th., Stechemesser, H. and Töpfer, E., "Investigations of the collision process between particles and gas bubbles in flotation: a theoretical analysis", Int. J. Miner. Process, Vol. 27, pp. 263-278, (1989).

47. Luerkens, D.W., Beddow, J.K. and Vetter, A.F., "Structure and morphology, the science of form applied to particle characterization", Powder Technology, Vol. 50, pp. 93-101, (1987).

48. Bonifazi, G. and Massacci, P., "Particle identification by image processing", KONA, No. 14, pp. 109-129, (1996).

49. Binnig, G., Quate, C.F., Geber, C., "Atomic force microscope", Phys. Rev. Lett., Vol. 56, No. 9, pp. 930-933, (1986).

50. Johnson, D.J., Miles, N.J., Hilal, N., "Quantification of particle-bubble interactions using atomic force microscopy: A review", Advances in Colloid and Interface Science, Vol. 127, pp. 67-81, (2006).

51. Preuss, M., Butt, H-J., "Measuring the Contact Angle of Individual Colloidal Particles", Journal of Colloid and Interface Science, Vol. 208, pp. 468-477, (1998).

52. Nguyen, A.V., Evans, G.M., Nalaskowski, J., Miller, J.D., "Hydrodynamic interaction between an air bubble and a particle: atomic force microscopy measurements", Experimental Thermal and Fluid Science, Vol. 28, pp. 387-394, (2004).

53. Butt, H.J., "A technique for measuring the force between a colloidal particle in water and a bubble", J. Colloid Interf. Sci., Vol. 166, pp. 109-117, (1994). 
54. Ducker, W.A., Xu, Z., Israelachvili, J.N., "Measurements of hydrophobic and DLVO forces in bubble-surface interactions in aqueous solutions", Langmuir, Vol. 10, pp. 3279-3289, (1994).

55. Fielden, M.L., Hayes, R.A., Ralston, J., "Surface and capillary forces affecting air bubble-particle interactions in aqueous electrolyte", Langmuir, Vol. 12, pp. 3721-3727, (1996).

56. Preuss, M., Butt, H.J., "Direct measurement of forces between particles and bubbles", Int. J. Miner. Process., Vol. 56, pp. 99-115, (1999).

57. Chan, D.Y.C., Dagastine, R.R., White, L.R., "Forces between a rigid probe particle and a liquid interface $\mathrm{I}$. The repulsive case", J. Colloid Interf. Sci., Vol. 236, pp. 141-154, (2001).

58. Attard, P., Miklavcic, S.J., "Effective spring constant of bubbles and droplets", Langmuir, Vol. 17, pp. 8217-8223, (2001).

59. Goetzinger, M. Peukert, W., "Dispersive forces of particle-surface interactions: direct AFM measurements and modeling", Powder Technology, Vol. 130, pp. 102- 109, (2003).

60. Li, T., Park, K., "Fractal analysis of pharmaceutical particles by atomic force microscopy", Pharm. Res., Vol. 15, No. 8, pp. 1222-1232, Aug., (1998).

61. Gladyszewski, G., Jaouen, C., Declemy, A., Girard, J.C., Guerin, P., "Ion-assisted deposition of $\mathrm{Ag}(001) / \mathrm{Fe}(001)$ multilayers: interface roughness", Thin Solid Films, Vol. 319, pp. 44-48, (1998).

62. Hazra, S., Pal, S., Kundu, S., Sanyal, M.K., "Scanning probe microscopy and Xray studies of confined metal films", Applied Surface Science, Vol. 182, pp. 244250, (2001).

63. Gwaze, P., Annegarn, H.J., Huth, J., Helas, G., "Comparison of particle sizes determined with impactor, AFM and SEM", Atmospheric Research, Vol. 86, pp. 93-104, (2007).

64. Brown, G.J., Miles, N.J. and Hall, S.T., "Fractal assessment of finely ground limestone for flue gas desulphurization", Minerals Engineering, Vol. 7, No. 8, pp. 1057-1067, (1994).

65. Frances, C., Bolay, N.L., Belaroui, K. and Pons, M.N., "Particle morphology of ground gibbsite in different grinding environments ", Int. J. Mineral Process., Vol. 61, pp. 41-56, (2001).

66. Ocepek, D., Strazisar, J. and Kanduti-Sumej, B., "Comminution and surface properties of minerals", $7^{\text {th }}$ European Symposium Comminution, Schonert, K. (Ed.), Clausthal, Zellerfeld, BRD, pp. 273-282, March, (1990).

67. Apel-Paz, M., Marmur, A., "Spreading of liquids on rough surfaces", Colloids and Surfaces A: Physicochemical and Engineering Aspects, Vol. 146, pp. 273-279, (1999).

68. Hazlett, R.D., "Fractal applications: wettability and contact angle", Journal of Colloid and Interface Science, Vol. 137, No. 2, pp. 527-533, July, (1990).

69. Hazlett, R.D., "On surface roughness effects in wetting phenomena", Contact Angle, Wttability and Adhesion, Mittal, K.L. (Ed.), VSP, pp. 173-181, (1993).

70. Onda, T., Shibuichi, S., Satoh, N., Tsujii, K., "Super-water-repellent fractal surfaces", Langmuir, Vol. 12, No. 9, pp. 2125-2127, May, (1996). 
71. Meirona, T.S., Marmurb, A. Saguy, I.S., "Contact angle measurement on rough surfaces", Journal of Colloid and Interface Science, Vol. 274, Issue 2, pp. 637644, June, (2004).

72. Ramesh, R. and Somasundaran, P., "Centrifugal immersion technique for characterizing the wettability of coal particles", J. of Colloid and Interface Sci., Vol. 139, No. 1, pp. 291-294, (1990).

73. Wotruba, H., "Untersuchungen zur Flotativen Trennung von Mikrolith und Zirkon unter besonderer Berücksichtigung des Kornformeinflusses", Ph.D. Thesis, Fakultät für Bergbau, Aachen, Germany, (1991).

74. Kaye, B.H., "Katastrophen im Labor", Physik im Unserer Zeit, Vol. 25, pp. 2935, (1994).

75. Nguyen, A.V., Schulze, H.J., Ralston, J., "Elementary steps in particle-bubble attachment", Int. J. Miner. Process, Vol. 51, pp. 183-195, (1997).

76. Nguyen, A.V., Stechemesser, H., Zobel, G., Schulze, H.J., "Order of three-phase (solid-liquid-gas) contact line tension as probed by simulation of three-phase contact line expansion on small hydrophobic spheres", J. of Colloid and Interface Sci., Vol. 187, pp. 547-550, (1997).

\section{دراسة تأثير عمليات التكسير و الطحن على شكل و خشونة سطح الحبيبات و علاقتهما بعمليات التعويم بالهواء: بحث مرجعى}

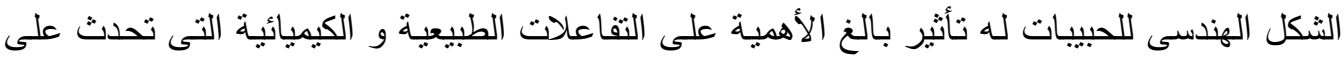
سطح الحبيبة خلال العطليات التكنولوجية. من هنا تتضح أهمية التحديد الهندسى الدقيق لثكل الحبيبات بإستخدام المتغيرات الكمية (القيم العددية) و التى أمكن تحديدها بسهولة فى السنوات الأخيرة نتيجة لتطور تكنولوجيا تحليل الصورة بالكمبيوتر .

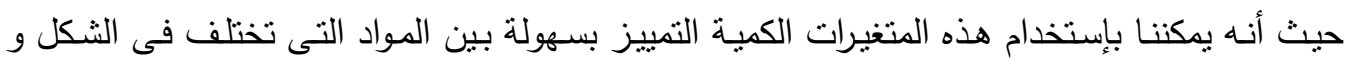
خشونة السطح و الناتجة عن عمليات تكسير و طحن مختلفة و كذلك ربط هذا الإختلاف بالعطيات

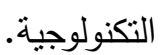

السمات الرئيسية المستخدمة لتمييز الحبيبات هى حجم الحبيبة، شكلها و خشونة سطحها. و يعتبر الثكل و خشونة السطح من المتغيرات المهمة التى يمكن إستخدامها فى التتبؤ بسلوك الحبيبات منفردة فئه

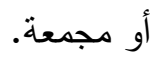

هذه المتغيرات مهمـة بصورة كبيرة للصناعات التى تتعامل مـع المواد المختلفة عندما تكون بأحجـام

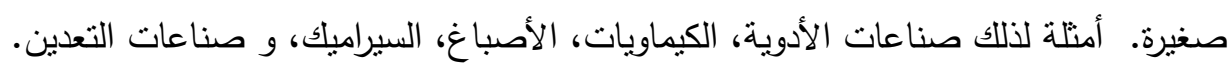


لذا فإن البحث يفند الطرق المختلفة المستخدمة لتحديد شكل و خشـونة سطح الحبيبـة و ربط هذه

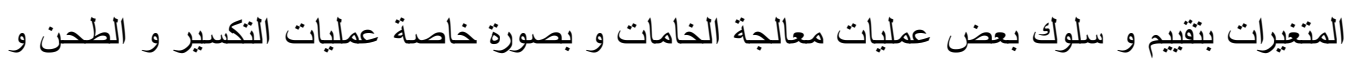
كذلك عمليات التعويم بالهواء. خشونة سطح الحبيبة تؤثر بصورة كبيرة على العمليات الأساسية التى تؤدى إلى إلتصاق الحبيبة بالفقاعة

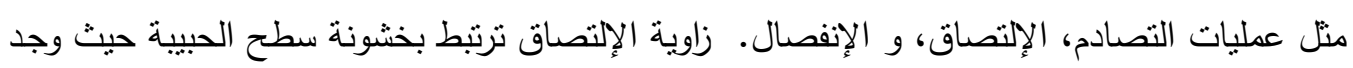

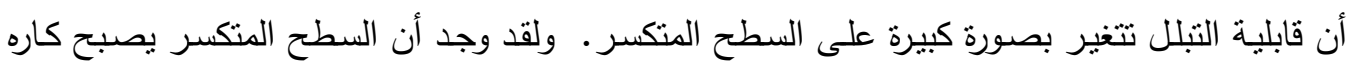

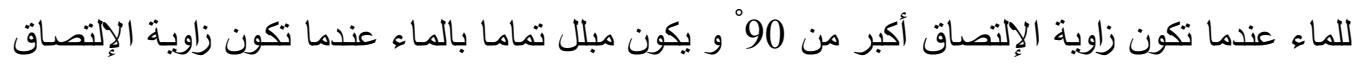
أقل من 90ْ.

عملية الطحن الجاف ينتج عنها أسطح خشنة نسبيا مع تواجد واضح لعيوب البنية الدقيقة بينما عملية الطحن الرطب ينتج عنها أسطح أنعم و أنظف. و لقد وجد أن العينات التى تم الحصول عليها بالطدن الجاف ينتج عنها معدلات تعويم أسرع و أكبر النها عن مثيلاتها التى نم الحصول عليها بالطحن الرطب.

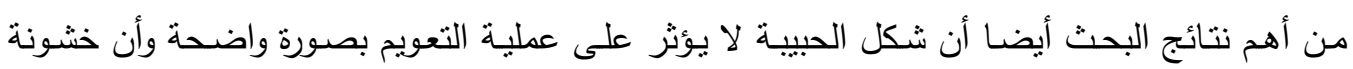

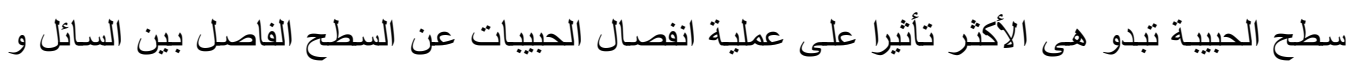
الفقاعة وكذلك علي عائد عملية التعويم. 